\title{
Estimativa do Tamanho Amostral em Medidas Repetidas de Circunferência Escrotal de Bovinos Nelore
}

\section{Alfredo Ribeiro de Freitas ${ }^{1,2}$, Antônio Emídio Dias Feliciano da Silva ${ }^{1,2}$, Marina Unanian ${ }^{1,2}$}

RESUMO - O objetivo deste trabalho foi estimar o tamanho amostral mínimo (n) de animais para se detectar diferença mínima significativa $(\Delta)$ entre avaliações mensais repetidas da circunferência escrotal de animais da raça Nelore, dos 10 aos 30 meses de idade, consideradas como 21 condições de avaliação ou medidas repetidas (tratamentos). O valor de n que permite detectar a $\Delta$ entre médias, quando cada indivíduo é avaliado para $\mathrm{t}>2$ tratamentos, foi obtido por meio de um programa desenvolvido no "Statistical Analysis System" - SAS, considerandose o modelo de distribuição normal $t$-variada $(\mathrm{t}=21)$ com vetor de média zero e matriz de covariância $\mathrm{S}$, estatística e distribuição $\mathrm{F}$ com parâmetro de não centralidade $\delta^{2} \Delta$. Diferentes níveis de erros do tipo I $(\alpha)$, potência do teste $(1-\beta)$, correlação mínima entre as medidas repetidas $\left(\rho_{\text {min }}\right)$ e $\Delta$ foram considerados. As estimativas de $\boldsymbol{n}$ variaram de 4 a 299 , sendo mais influenciadas por variações na $\Delta$.

Palavras-chave: distribuição $F$ e parâmetro de não centralidade $\delta^{2} \Delta$, erro do tipo $\mathrm{I}(\alpha)$, potência do teste (1- $\left.\beta\right)$, medidas repetidas, simulação

\section{Estimate of Sample Size in Repeated Measurements of Scrotal Circumference of Nellore Cattle}

ABSTRACT - The objective of this work was to estimate minimum sample size (n) of animals to detect significant differences $(\Delta)$ among monthly replicate evaluations of scrotal circumference from animals of the Nellore breed, from 10 to 30 months of age, considered as 21 evaluation conditions or repeated measurements (treatments). The value of $\mathrm{n}$ that allows to detect the $\Delta$ among means when each subject is observed for $\mathrm{t}>2$ treatments, was obtained by means of the Statistical Analysis System - SAS developed program, the $\mathrm{t}$-variate normal distribution $(\mathrm{t}=21)$, as a vector of zero mean and $\mathrm{S}$ covariate matrix, Hotelling $\mathrm{T}^{2}$ statistics and $\mathrm{F}$-distribution with noncentrality parameter of $\delta^{2} \Delta$. Different levels of type I error $(\alpha)$, power of the test $(1-\beta)$, minimum correlation among repeated measurements $\left(\rho_{\min }\right)$ and $\Delta$ were considered. The $\boldsymbol{n}$ estimates ranged from 4 to 299 , being mostly affected by changes in the $\Delta$.

Key Words: F distribution with noncentrality parameter $\delta^{2} \Delta$, type I error $(\alpha)$, power of the test $(1-\beta)$, repeated measurements, simulation

\section{Introdução}

O uso de métodos que consideram medidas repetidas avaliadas na mesma unidade experimental tem recebido mais atenção nos últimos anos, principalmente, devido a sua eficiência em relação a delineamentos tradicionais, como, por exemplo, os de tratamentos aleatorizados em blocos com parcelas divididas ou subdivididas (VONESH e SCHORK, 1986; SAS, 1993). GEARY (1989) realizou excelente abordagem teórica desses métodos, com ênfase na modelagem da estrutura de covariância e estimativas dos parâmetros por máxima verossimilhança e razão de verossimilhança, como critérios para escolha de modelos.

Um exemplo de aplicação destes métodos na agropecuária consiste na avaliação da circunferência escrotal (CE) de bovinos de corte a partir dos 10 meses de idade. Esta característica possui inúmeras qualidades como critério de seleção de machos: fácil mensuração, herdabilidade de média a alta magnitude e correlação alta com características produtivas e reprodutivas, tanto de machos (capacidade de serviço, libido, nível de testosterona, volume e qualidade do sêmen) quanto de fêmeas (precocidade à puberdade, atividade ovariana) (LUNSTRA et al., 1988; FELICIANO SILVA et al., 1991; ELER et al., 1996; PRATT et al., 1991; e PINTO, 1996).

Apesar da importância da CE para propósitos de reprodução e melhoramento de bovinos, praticamente inexistem na literatura estudos sobre a determinação do tamanho amostral mínimo que assegure inferências confiáveis e métodos de análise mais abrangentes como os de medidas repetidas.

O objetivo deste trabalho foi estimar o número de animais necessário para se detectar diferença mínima significativa entre médias, considerando-se avaliações mensais de CE de bovinos da raça Nelore, dos 10 aos 30 meses de idade, podendo-se escolher a precisão com que se deseja testar os tratamentos. 


\section{Material e Métodos}

\section{Obtenção dos dados}

Foram utilizados dados de CE de 30 animais da raça Nelore, obtidos no período de 1987 a 1988, em Campo Grande, MS, sendo consideradas 21 avaliações por animal, dos 10 aos 30 meses de idade; a CE foi avaliada nos dois testículos por meio de fita métrica milimetrada, no ponto de maior diâmetro das bolsas escrotais.

\section{Método de análise}

Os dados deCE foram analisados pormeio do modelo:

$\mathrm{y}_{\mathrm{i}}=\mu+\varepsilon_{\mathrm{i}} \quad(\mathrm{i}=1, \ldots, \mathrm{n}), \varepsilon_{\mathrm{i}} \sim \operatorname{IID~N}_{\mathrm{t}}(0, \Sigma)$

em que $y_{i^{\prime}}=\left(y_{i 1}, \ldots, y_{i t}\right)$ é o vetor de resposta do i-ésimo animal nos t tempos sucessivos de avaliação; $\mu^{\prime}=\left(\mu_{1}, \ldots, \mu_{t}\right)$, o vetor de resposta média no tempot; e $\varepsilon_{i}$, o erro experimental de distribuição normal $t$ variada $(\mathrm{t}=21)$, identicamente distribuído, com vetor de média zero e matriz de covariâncias $\sum$.

$O$ teste para aceitar ou rejeitar a hipótese nula Ho: $\mu_{1}=\ldots .=\mu_{\mathrm{t}}$, ou seja, a igualdade do comportamento do perfil médio ao longo do tempo é a estatística $\mathrm{T}^{2}$ de Hotelling dada por:

$$
\mathrm{T}^{2}=\mathrm{n} \bar{Y}^{\prime} \mathbf{C}\left(\mathbf{C}^{\prime} \mathbf{S C}\right)^{-1} \mathbf{C}^{\prime} \bar{Y}
$$

em que

$\bar{Y}=\sum_{i=1}^{n} \mathrm{y}_{\mathrm{i}} / \mathrm{n} \quad$ é $\quad$ o vetor de média; $\mathrm{S}=(\mathrm{n}-1)^{-1} \sum_{i=1}^{n}\left(\mathrm{y}_{\mathrm{i}}-\bar{Y}\right)\left(\mathrm{y}_{\mathrm{i}}-\bar{Y}\right)^{\prime}$, a matriz de covariância amostral, positiva definida; e $\mathrm{C}$, qualquer matriz de contraste ortogonal ( $\mathrm{t}-1) \mathrm{x}$ (VONESH e SCHORK, 1986). A estatística $\mathrm{T}^{2}$ possui $(\mathrm{t}-1)$ e (n - 1) graus de liberdade e parâmetro de não centralidade $\delta^{2}=n \mu^{\prime} \mathbf{C}\left(\mathbf{C}^{\prime} \boldsymbol{\Sigma} \mathbf{C}\right)-\mathbf{1} \mathbf{C}^{\prime} \mu$.

Sob Ho verdadeira, obtém-se:

$\mathrm{F}=(\mathrm{n}-\mathrm{t}+1)[(\mathrm{n}-1)(\mathrm{t}-1)]^{-1} \mathrm{~T}^{2}$, que possui distribuição $\mathrm{F}$ com graus de liberdade $\mathrm{v}_{1}=(\mathrm{t}-1)$, $\mathrm{v}_{2}=(\mathrm{n}-\mathrm{t}+1)$ e parâmetro de não centralidade $\delta^{2}$. Para dado $\alpha$, rejeita-se Ho se $\mathrm{F}>\mathrm{F}\left(\mathrm{v}_{1}, \mathrm{v}_{2} ; \delta^{2}\right)$.

Uma vez que não se conhecem todos os valores de $\mu$ e $\Sigma$ para os quais se deseja aceitar ou rejeitar Ho, especificou-se, entre quaisquer duas médias, diferença mínima significativa $(\Delta)$, cuja significância estatística se deseja comprovar em nível a de probabilidade e potência do teste (1-b), sujeito à restrição $\left|\mu_{\mathrm{j}}-\mu_{\kappa}\right|>\Delta$, para algum $\mathrm{j} \neq \mathrm{k}$, (SCHEFFÉ, 1959).

$\mathrm{O}$ valor mínimo de $\delta^{2}$ sujeito à restrição $\left|\mu_{\mathrm{j}}-\mu_{\kappa}\right|=$ $\Delta$, definido por $\delta^{2} \Delta$ é igual a $\mathrm{n} \Delta^{2} / \max \mathrm{j}<\mathrm{k}\left\{\sigma^{2}{ }_{\mathrm{j}}+\sigma_{\mathrm{k}}^{2}\right.$ - $\left.2 \sigma_{\mathrm{jk}}\right\}$, em que $\sigma_{\mathrm{j}}^{2}$ e $\sigma_{\mathrm{k}}^{2}(\mathrm{j}<\mathrm{k})$ são as variâncias e $\sigma_{\mathrm{jk}}$, a covariância, associadas às características $\mathrm{j}$ e k, respectivamente. Considerando-se $\Sigma$, qualquer matriz de variância-covariância, positiva definida, pode ser demonstrado (VONESH e SCHORK, 1986) que a expressão $n \Delta^{2} /\left[\left(2 \sigma_{\text {max }}^{2}\left(1-\rho_{\text {min }}\right)\right]<\delta^{2} \Delta\right.$, em que $\rho_{\text {min }}$ é o menor coeficiente de correlação entre medidas repetidas, $\sigma_{\max }^{2}=\operatorname{máximo}\left(\sigma_{\mathrm{j}}^{2}\right)$ e ainda $\Delta$ medido em unidades de $\sigma_{\text {max }}$, é apropriada para estimar o tamanho amostral $\boldsymbol{n}$.

As estimativas de $\boldsymbol{n}$, para $\mathrm{t}>2$ tratamentos, utilizando-se essa expressão e ainda $\mathrm{v}_{1}, \mathrm{v}_{2}, \alpha$ e potência do teste $(1-\beta)$, foram obtidas iterativamente por meio do programa SAS que utiliza as funções FPROB e FINV (HARDISON et al., 1983; VONESH e SCHORK, 1986 ). O programa avalia a integral abaixo, em que $\boldsymbol{f}_{\boldsymbol{F}}$ denota a função de densidade da distribuição F não-central e $\mathbf{F}_{\boldsymbol{\alpha}}\left(\mathbf{v}_{\mathbf{1}}, \mathbf{v}_{\mathbf{2}}\right)$ representa a porcentagem $100(1-\alpha)$ dessa distribuição:

$$
1 \text { - } \beta(n)=\int_{F_{\alpha}\left(v_{1}, v_{2}\right)}^{\infty} f_{F}\left(x, v_{1}, v_{2}, \delta_{\Delta}^{2}\right) d x,
$$

O valor de n é estimado da desigualdade 1 - $\beta(n)$ $>1$ - $\beta$; considerando $\alpha(0,01$ a 0,05$)$, potência do teste $(0,95$ e 0,90$), \rho_{\min }$ assumindo os valores de 0,$1 ; 0,3$; 0,$5 ; 0,7$; e 0,9 e $\Delta$ os de $0,5 \sigma ; 1,0 \sigma$ e $1,5 \sigma ; 2,0 \sigma$ e $2,5 \sigma$, sendo $\sigma=\sigma_{\text {max }}^{2}$.

\section{Resultados e Discussão}

A partir da matriz de variância-covariância dos dados, positiva definida (Tabela 1), em que $\sigma_{\max }^{2}=4,3864$, o valor de $\mathrm{n}$ a ser estimado foi obtido em função da expressão $0,1139 n \Delta^{2} /\left(1-\rho_{\min }\right), v_{1}, v_{2}$, $\alpha$ e $\beta$. Por incluir $\rho$ min, esta expressão leva em conta o fato das correlações decrescentes entre medidas repetidas, à medida que o intervalo de tempo entre as observações aumenta; por considerar $\sigma_{\text {max }}^{2}$, deve-se considerar um fato comum em estudos de crescimento, ou seja, de que o efeito de um tratamento com o tempo está associado a incremento da variância. Essas propriedades, associadas ao fato de que a matriz de variância-covariância é definida positiva, asseguram confiabilidade maior nos valores de $\mathrm{n}$ (BROWNIE et al., 1990; CULLIS e McGILCHRIST, 1990).

A Figura 1 apresenta o crescimento típico da CE para bovinos da raça Nelore dos 10 aos 30 meses de idade. O desenvolvimento é crescente, porém, com incrementos menores, à medida que o animal fica mais velho. Este padrão de crescimento está de acordo com o obtido em bovinos avaliados em dife- 
Tabela 1- Matriz de variância-covariância amostral considerando 21 medidas repetidas da circunferência escrotal de animais da raça Nelore Table 1- Matrix of sample covariances considering 21 repeated measures of scrotal circumference from Nellore Cattle

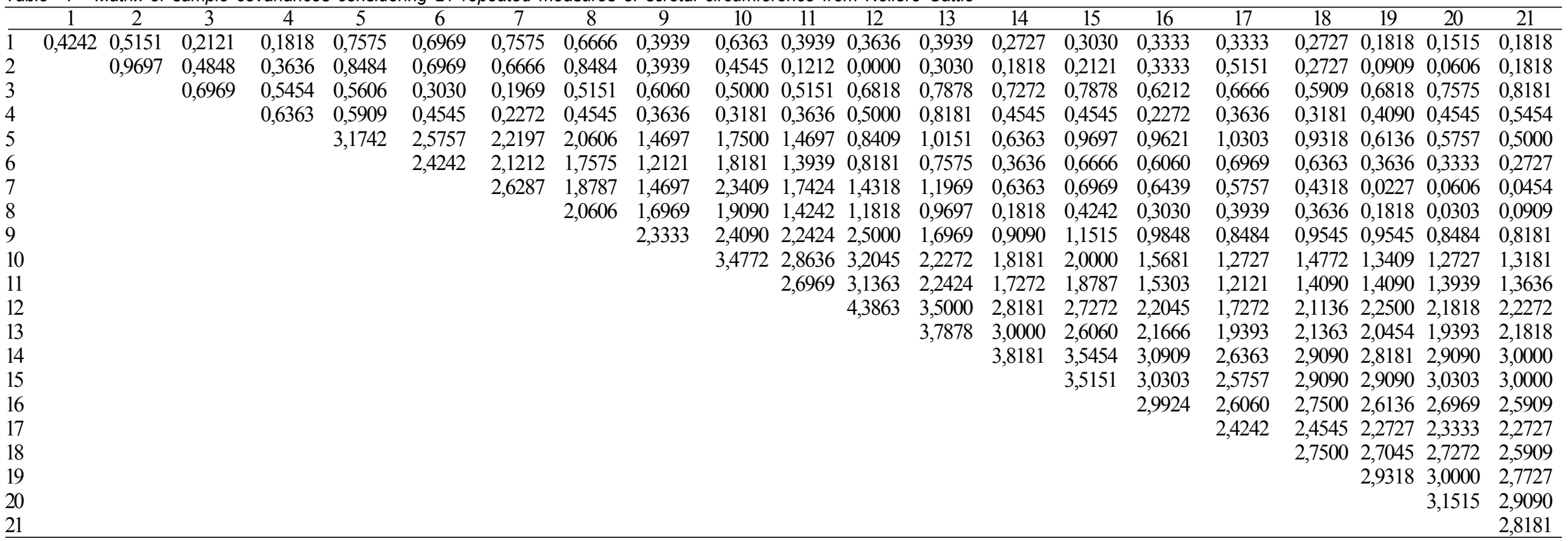




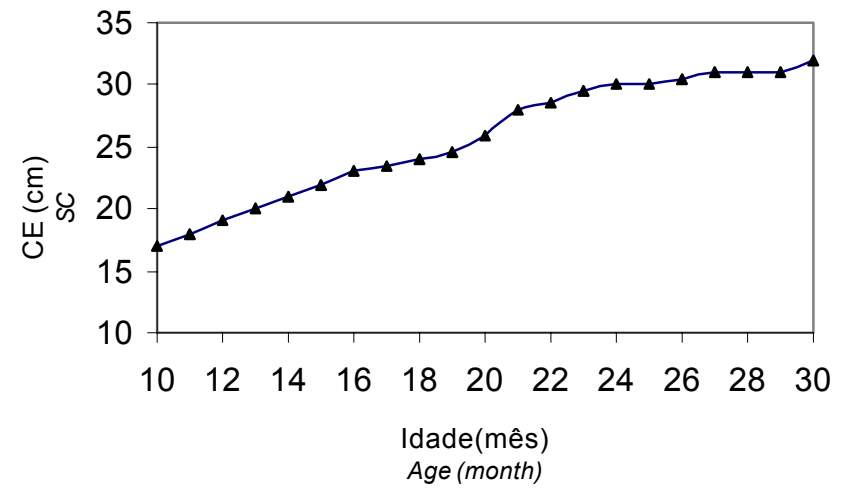

Figura 1 - Médias da circunferência escrotal (cm), dos 10 aos 30 meses de idade, obtidas de bovinos Nelore.

Figure 1 - Means of scrotal circumference from 10 to 30 months of age obtained from Nellore cattle.

rentes raças e locais (BOURDON e BRINKS, 1986; LUNSTRA et al., 1988; ALENCAR e VIEIRA, 1989; PRATT et al., 1991; QUIRINO et al., 1996; PINTO, 1996; e FREITAS et al., 1997).

Dentro deste período de estudo da CE, algumas considerações são apresentadas, sobre esta característica, para propósitos de reprodução e melhoramento:

a) A CE tem sido avaliada considerando a idade de 12 (MOURA e ERICKSON, 1996) ou 18 meses, ou intervalos de medidas repetidas, em que valores adjacentes são mais correlacionados. Alguns exemplos deste último tipo de avaliação são: dos 368 a 400 dias (KING et al., 1983), 205 e 365 dias (TOELLE e ROBISON, 1985), 608 a 852 dias (COULTER et al., 1987), 300 a 400 dias (LUNSTRA et al., 1988), 330 e 430 dias (KRIESE et al., 1991), cinco avaliações com intervalo de 28 dias pós-desmame (PRATT et al., 1991), 12, 18 e 24 meses (ALENCAR et al., 1993), 9, 18, 24 e 30 meses (QUIRINO et al., 1996) e 10 a 30 meses (FREITAS et al., 1997).

b) Em cada idade, a CE parece ser controlada por fatores fisiológicos diferentes: hormônios, até atingir a maturidade ( 24 meses), e o desenvolvimento corporal propriamente dito, que resulta em aumento de peso (até 30 meses). Entretanto, a partir dos 12 meses, o seu desenvolvimento sofre maior influência hormonal (TOELLE e ROBISON, 1985). Este fato sugere que a CE varia em função da fisiologia específica de cada idade. Como as variâncias genéticas aumentam ou reduzem segundo a idade do animal (URIBE, 1996), estimativas diferenciadas de herdabilidade $\left(\mathrm{h}^{2}\right)$ podem ser obtidas para a $\mathrm{CE}$ dentro do intervalo de 10 a 30 meses de idade.

c) Como o peso corporal varia consideravelmente para a mesma idade, é de maior interesse, para propósitos de seleção, estimar $\mathrm{h}^{2}$ da CE em função da idade e não do peso (LUNSTRA et al., 1988).

d) Os touros devem possuir valor mínimo da CE dentro de cada idade-raça para propósitos de seleção (COULTER et al., 1987); touros que possuem valores altos para CE quando adultos, ou seja, que excedem o valor mínimo da idade-raça a partir dos 21 meses de idade, porém, que apresentaram desenvolvimento testicular lento até este período, não devem ser usados em programas de melhoramento. Assim, é interessante fazer avaliações repetidas da $\mathrm{CE}$ dentro de cada idade-raça, para estimar curvas de crescimento mais realistas e explicar esta interação, a qual tem sido encontrada em bovinos (LUNSTRA et al. 1988; ALENCAR e VIEIRA, 1989; e ALENCAR et al., 1993).

e) As avaliações repetidas no mesmo animal, principalmente quando analisadas sob o enfoque de modelos de regressão aleatória "random regression models" (URIBE, 1996), apresentam qualidades importantes para o melhoramento, por várias razões: minimizam o erro de predição e consideram as oscilações de variâncias genéticas e residuais com o tempo, ou seja, permitem estimar herdabilidade diferenciada para cada idade, uma vez que as variâncias genéticas aumentam ou reduzem segundo a idade. Permitem ainda dividir a variância fenotípica em dois componentes, variância entre indivíduos, que mede as variâncias genética total e permanente e variância dentro de indivíduos, que mede diferenças temporárias de ambiente entre sucessivas avaliações (MANSOUR et al., 1991).

Tabela 2 - Amplitude do número de animais ulitizados para avaliação da circunferência escrotal dentro dos vários efeitos: ano, grupo genético, local, entre outros

Table 2 - Range of number of animals used for scrotal circumference evaluation considering several effects: year, genetic group, local, among others

\begin{tabular}{lc}
\hline $\begin{array}{l}\text { Autor } \\
\text { Author }\end{array}$ & $\begin{array}{c}\text { Efeito } \\
\text { Effect }\end{array}$ \\
\hline BRINKS etal., 1978 & 5 a 22 \\
KING etal., 1983 & 25 \\
WERREeBRINKS, 1986 & 3 a 81 \\
COULTER etal., 1987 & 80 a 3769 \\
LUSTRA etal., 1988 & 144 a 488 \\
SWANEPOEL et al., 1988 & 10 \\
FELICIANO SILVA et al., 1991 & 10 \\
PRATT et al., 1991 & 14 a 71 \\
ALENCAR etal, 1993 & 101 a 567 \\
MOURA e ERIKSON, 1996 & 24 \\
QUIRINO etal., 1996 & 16 a 254 \\
\hline
\end{tabular}


Rev. bras. zootec.

f) As afirmações de "a" até "e" justificam o uso do método de medidas repetidas em estudos da CE; assim, uma preocupação adicional é a determinação do tamanho amostral que possibilite obter inferências precisas utilizando-se este método, pois o valor de $n$ aumenta em função do número de avaliações (tratamentos) feitas no mesmo animal (Figuras 2 a 6 ). No entanto, na literatura consultada (Tabela 2), verificase que não existe preocupação sobre o tamanho amostral mínimo de indivíduos para obter estimativas precisas; porém, esta exigência parece ainda mais importante em situações em que se estimam também parâmetros genéticos por meio de métodos assintóticos, como os da máxima verossimilhança (ML) e ML restrita, que exigem amostra grande de indivíduos.

As Figuras 2 a 6 apresentam as estimativas de $n$ em função dos $\mathrm{t}$ tratamentos (2 a 21), $\alpha(0,01$ a 0,05$)$, potência do teste $(0,95$ e 0,90$)$, correlação entre medidas repetidas de 0,1 a 0,9 e de $\Delta$, assumindo os valores $0,5 \sigma$ a $2,5 \sigma$. As Figuras estão diferenciadas de acordo com o valor de $\Delta$; Figuras $2(\Delta=0,5 \sigma) ; 3(\Delta=1,0 \sigma)$; $4(\Delta=1,5 \sigma), 5(\Delta=2,0 \sigma)$ e $6(\Delta=2,5 \sigma), \alpha=0,01 \mathrm{e}$ $\beta=0,05$ (A), $\alpha=0,01$ e $\beta=0,10$ (B), $\alpha=0,05$ e $\beta=0,05$
(C), $\alpha=0,05$ e $\beta=0,10(\Delta)$. As cinco linhas das Figuras, em ordem decrescente de $n$, indicam valores de $\rho_{\text {min }}$ de 0,$1 ; 0,3 ; 0,5 ; 0,7$; e 0,9 , respectivamente.

Considerando correlação mínima entre medidas repetidas de $0,1\left(\rho_{\min }=0,1\right)$, para se detectar diferença mínima significativa de $\Delta=0,5 \sigma$ entre duas médias e $\alpha=0,01$, há necessidade de 111 e 132 indivíduos (duas avaliações) até 263 a 299 (21 avaliações); para potência do teste de 0,90 e 0,95 , respectivamente (Figura 2 A,B). Mantendo-se esta situação, porém considerando-se $\rho_{\min }=0,9$, há necessidade de 16 a 95 indivíduos, o que evidencia que o tamanho da amostra diminui quando $\rho$ aumenta. Analisando-se ainda a Figura 2, verifica-se que para $\alpha=0,05$ são necessários 96 a $238(1-\beta=0,05)$ e 78 a 205 $(1-\beta=0,90)$ indivíduos para se detectar diferença significativa entre médias, quando se considera amplitude de 2 até 21 avaliações. Observa-se, dentro de cada valor de $\Delta$, que o tamanho da amostra é pouco influenciado por valores de $\alpha, \beta$, para $\rho_{\min }>0,5$.

A Tabela 3 apresenta a amplitude dos valores de $\mathrm{n}$, considerando-se as 21 condições de avaliação dentro de cada combinação de $\alpha, \beta$ e $\rho_{\text {min }}$. Para

Tabela 3 - Amplitude dos valores de $\mathrm{n}$ considerando 21 medidas repetidas de circunferência escrotal de bovinos da raça Nelore

Table 3 - $\quad$ Range of values of $n$ considering 21 repeated measures of the scrotal circumference of Nellore cattle

\begin{tabular}{|c|c|c|c|c|c|c|}
\hline$\alpha$ & $\beta$ & $\rho=0,1$ & $\rho=0,3$ & $\rho=0,5$ & $\rho=0,7$ & $\rho=0,9$ \\
\hline \multicolumn{7}{|c|}{$\Delta=0,5 \sigma$} \\
\hline 0,01 & 0,05 & 132 a 299 & 104 a 237 & 75 a 175 & 47 a 113 & 18 a 52 \\
\hline 0,01 & 0,10 & 111 a 263 & 87 a 209 & 63 a 155 & $40 \mathrm{a} 101$ & $16 \mathrm{a} 48$ \\
\hline 0,05 & 0,05 & 96 a 238 & 75 a 189 & 54 a 140 & 34 a 91 & 13 a 43 \\
\hline 0,05 & 0,10 & 78 a 205 & 61 a 163 & 44 a 121 & 28 a 80 & $11 \mathrm{a} 40$ \\
\hline \multicolumn{7}{|c|}{$\Delta=1,0 \sigma$} \\
\hline 0,01 & 0,05 & 36 a 90 & 29 a 75 & 22 a 60 & 15 a 45 & 8 a 31 \\
\hline 0,01 & 0,10 & 31 a 81 & 25 a 68 & 19 a 55 & 13 a 42 & 7 a 29 \\
\hline 0,05 & 0,05 & 26 a 73 & 21 a 61 & 16 a 49 & 10 a 38 & 5 a 27 \\
\hline 0,05 & 0,10 & 21 a 65 & 17 a 55 & 13 a 45 & 9 a 35 & 5 a 26 \\
\hline \multicolumn{7}{|c|}{$\Delta=1,5 \sigma$} \\
\hline 0,01 & 0,05 & 18 a 52 & 15 a 46 & 12 a 39 & 9 a 33 & 6 a 27 \\
\hline 0,01 & 0,10 & 16 a 48 & 13 a 43 & 11 a 37 & 8 a 31 & 5 a 26 \\
\hline 0,05 & 0,05 & 13 a 43 & 11 a 38 & 8 a 33 & 6 a 29 & 4 a 24 \\
\hline 0,05 & 0,10 & 11 a 40 & 9 a 36 & 7 a 32 & 6 a 28 & 4 a 24 \\
\hline \multicolumn{7}{|c|}{$\Delta=2,0 \sigma$} \\
\hline 0,01 & 0,05 & 12 a 39 & 10 a 36 & 8 a 32 & 7 a 29 & 5 a 25 \\
\hline 0,01 & 0,10 & 11 a 37 & 9 a 34 & 8 a 31 & 6 a 28 & 4 a 25 \\
\hline 0,05 & 0,05 & 9 a 34 & 7 a 31 & 6 a 28 & 5 a 26 & 4 a 23 \\
\hline 0,05 & 0,10 & 7 a 32 & 6 a 29 & 5 a 27 & 4 a 28 & 3 a 23 \\
\hline \multicolumn{7}{|c|}{$\Delta=2,5 \sigma$} \\
\hline 0,01 & 0,05 & 9 a 34 & 8 a 31 & 7 a 29 & 6 a 27 & 4 a 24 \\
\hline 0,01 & 0,10 & 8 a 32 & 7 a 30 & 6 a 28 & 5 a 26 & 4 a 29 \\
\hline 0,05 & 0,05 & 7 a 29 & 6 a 28 & 5 a 26 & 4 a 25 & 4 a 23 \\
\hline 0,05 & 0,10 & 6 a 28 & 5 a 27 & 5 a 25 & 4 a 24 & 4 a 23 \\
\hline
\end{tabular}


FREITAS et al.
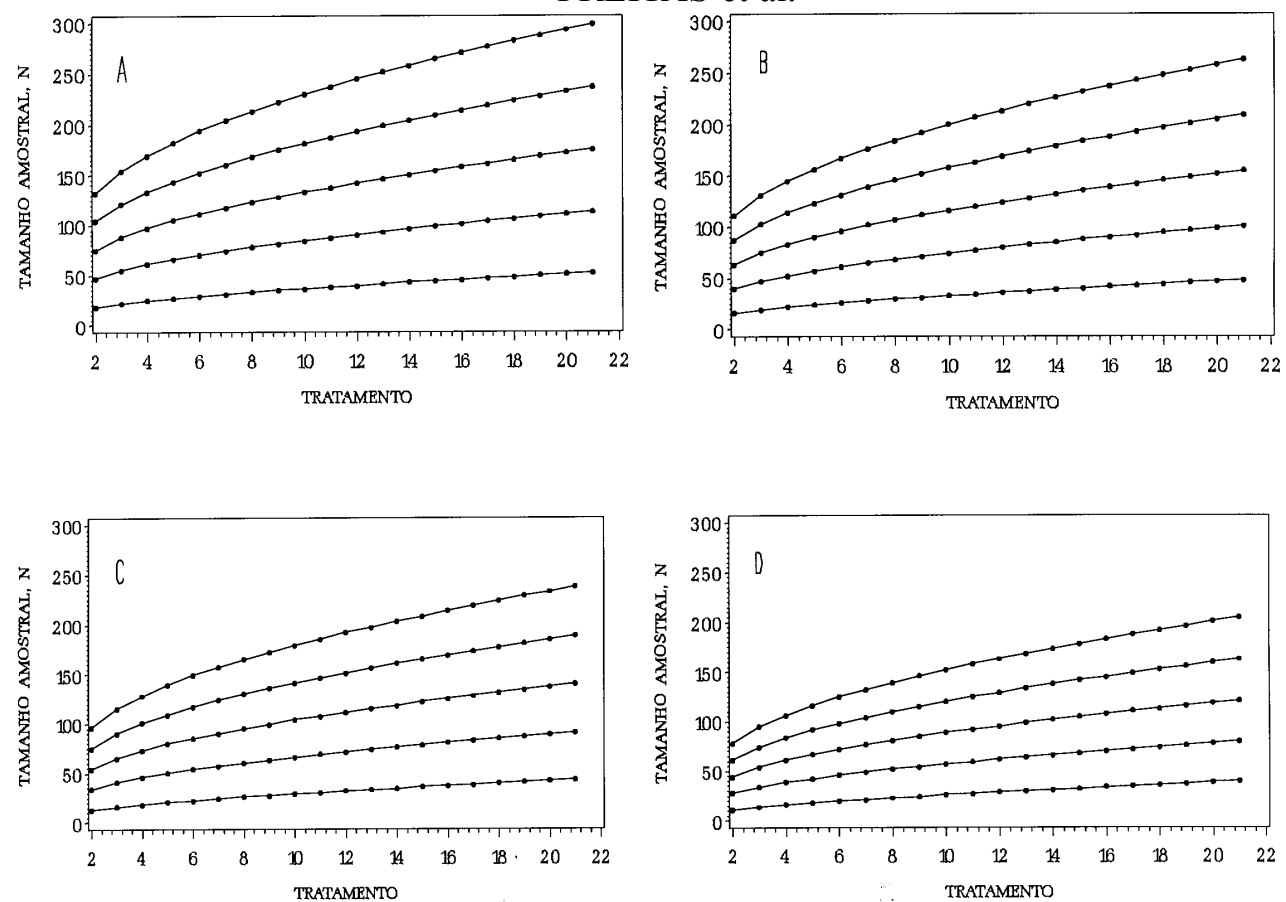

Figura 2 - Estimativas do tamanho amostral $(\mathrm{N})$ para $\Delta=0,5 \sigma, \alpha=0,01$ e $\beta=0,05(\mathrm{~A}), \alpha=0,01$ e $\beta=0,10$ (B), $\alpha=0,05$ e $\beta=0,05$ (C), $\alpha=0,05$ e $\beta=0,10$ (D). As linhas, em ordem decrescente de $N$, indicam valores acima de $\rho_{\text {min }}$ de 0,$1 ; 0,3 ; 0,5$; 0,7 ; e 0,9 , respectivamente.

Figure 2 - Estimates of sample size $(N)$ for $\Delta=.5 \sigma ; \alpha=.01$ and $\beta=.05(A), \alpha=.01$ and $\beta=.10(B), \alpha=.05$ and $\beta=.05$ (C), $\alpha=.05$ and $\beta=.10$ (D). The rows, in decreasing valies of $n$, indicate values for $\rho_{\min }$ de .1, .3, .5, .7, and .9, respectively.
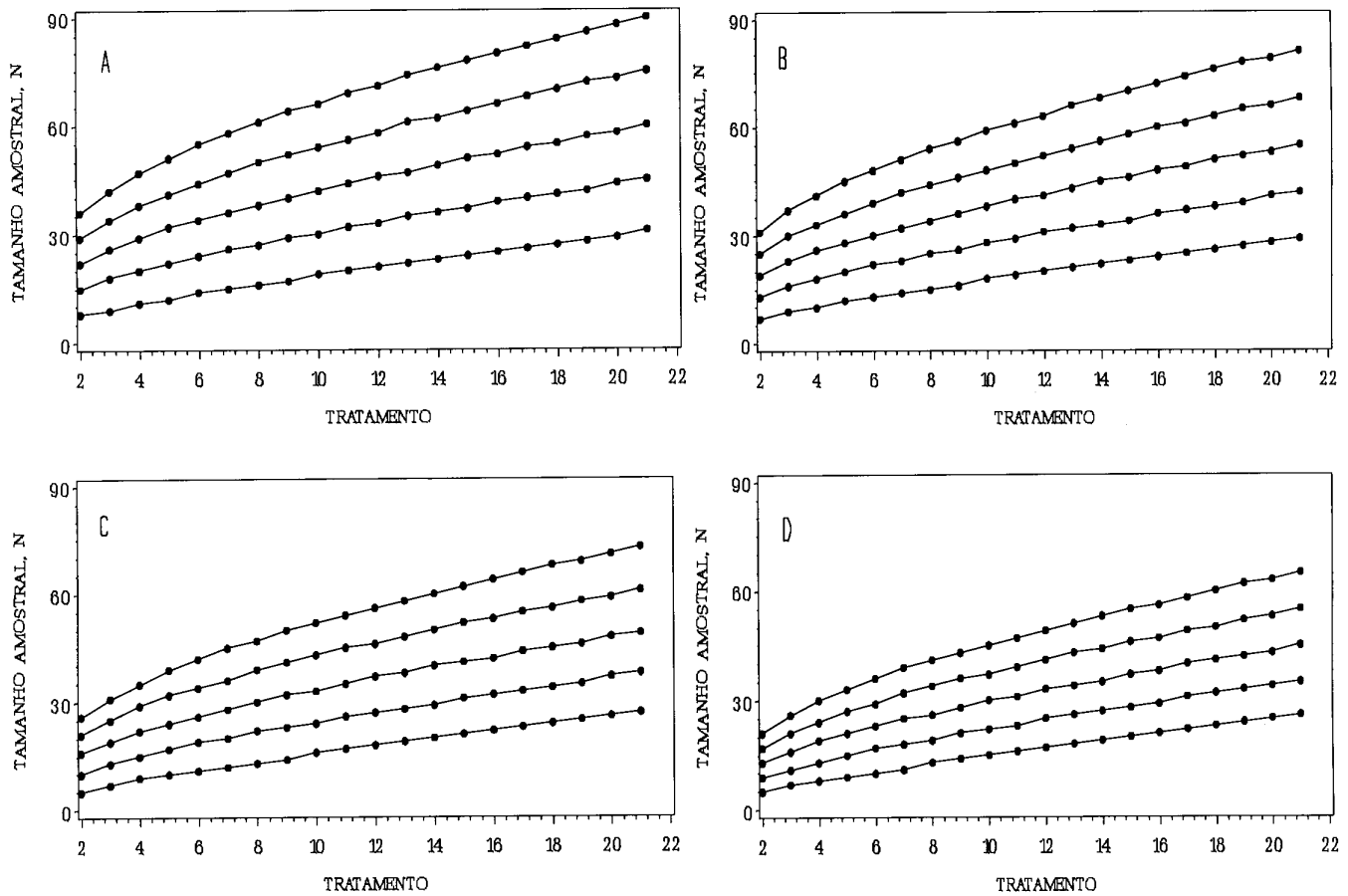

Figura 3 - Estimativas do tamanho amostral $(\mathrm{N})$ para $\Delta=1,0 \sigma, \alpha=0,01$ e $\beta=0,05(\mathrm{~A}), \alpha=0,01$ e $\beta=0,10$ (B), $\alpha=0,05$ e $\beta=0,05$ (C), $\alpha=0,05$ e $\beta=0,10$ (D). As linhas, em ordem decrescente de $N$, indicam valores acima de $\rho_{\min }$ de 0,$1 ; 0,3 ; 0,5$; 0,7 ; e 0,9 , respectivamente.

Figure 3 - Estimates of sample size $(N)$ for $\Delta=1.0 \sigma ; \alpha=.01$ and $\beta=.05(A), \alpha=.01$ and $\beta=.10(B), \alpha=.05$ and $\beta=.05$ (C), $\alpha=.05$ and $\beta=.10$ (D). The rows, in decreasing values of $n$, indicate values for $\rho_{\min }$ de .1, .3, .5, .7, and .9, respectively. 

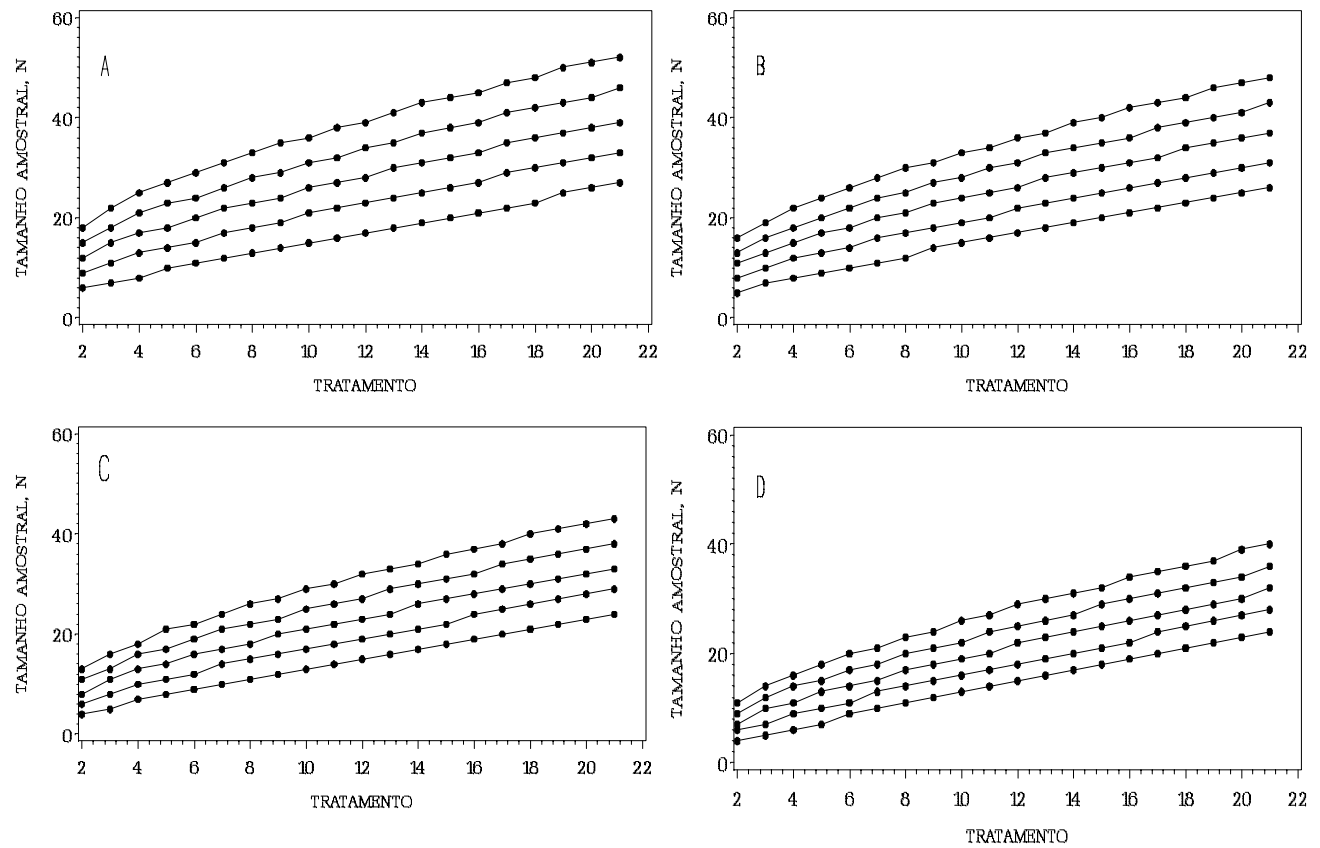

Figura 4 - Estimativas do tamanho amostral (N) para $\Delta=1,5 \sigma, \alpha=0,01$ e $\beta=0,05$ (A), $\alpha=0,01$ e $\beta=0,10$ (B), $\alpha=0,05$ e $\beta=0,05$ (C), $\alpha=0,05$ e $\beta=0,10$ (D). As linhas, em ordem decrescente de $N$, indicam valores acima de $\rho_{\min }$ de 0,$1 ; 0,3 ; 0,5$; 0,$7 ;$ e 0,9 , respectivamente.

Figure 4 - Estimates of sample size $(N)$ for $\Delta=1.5 \sigma, \alpha=.01$ and $\beta=.05(A), \alpha=.01$ and $\beta=.10(B), \alpha=0.05$ and $\beta=.05(C), \alpha=.05$ and $\beta=$ .10 (D). The rows, in decreasing values of $n$, indicate values for $\rho_{\min }$ de $.1, .3, .5, .7$, and .9 , respectively.
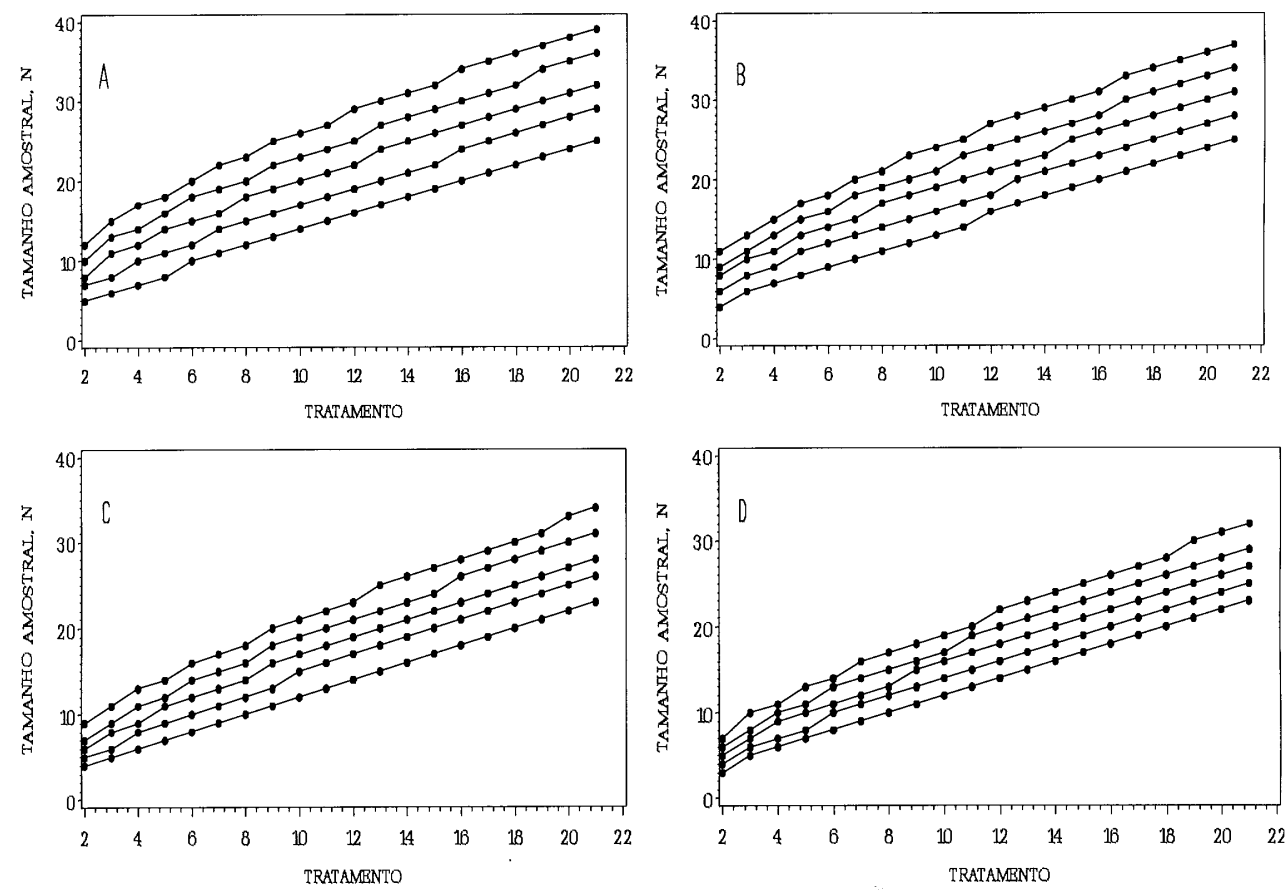

Figura 5 - Estimativas do tamanho amostral (N) para $\Delta=2,0 \sigma, \alpha=0,01$ e $\beta=0,05$ (A), $\alpha=0,01$ e $\beta=0,10$ (B), $\alpha=0,05$ e $\beta=0,05$ (C), $\alpha=0,05$ e $\beta=0,10$ (D). As linhas, em ordem decrescente de $N$, indicam valores acima de $\rho_{\min }$ de 0,$1 ; 0,3 ; 0,5$; 0,7 ; e 0,9 , respectivamente.

Figure 5 - Estimates of sample size (N) for $\Delta=2.0 \sigma, \alpha=.01$ and $\beta=.05$ (A), $\alpha=.01$ and $\beta=.10$ (B), $\alpha=.05$ and $\beta=.05$ (C), $\alpha=.05$ and $\beta=.10(D)$. The rows, in decreasing values of $n$, indicate values for $\rho_{\min }$ de .1, .3, .5, .7, and .9, respectively. 

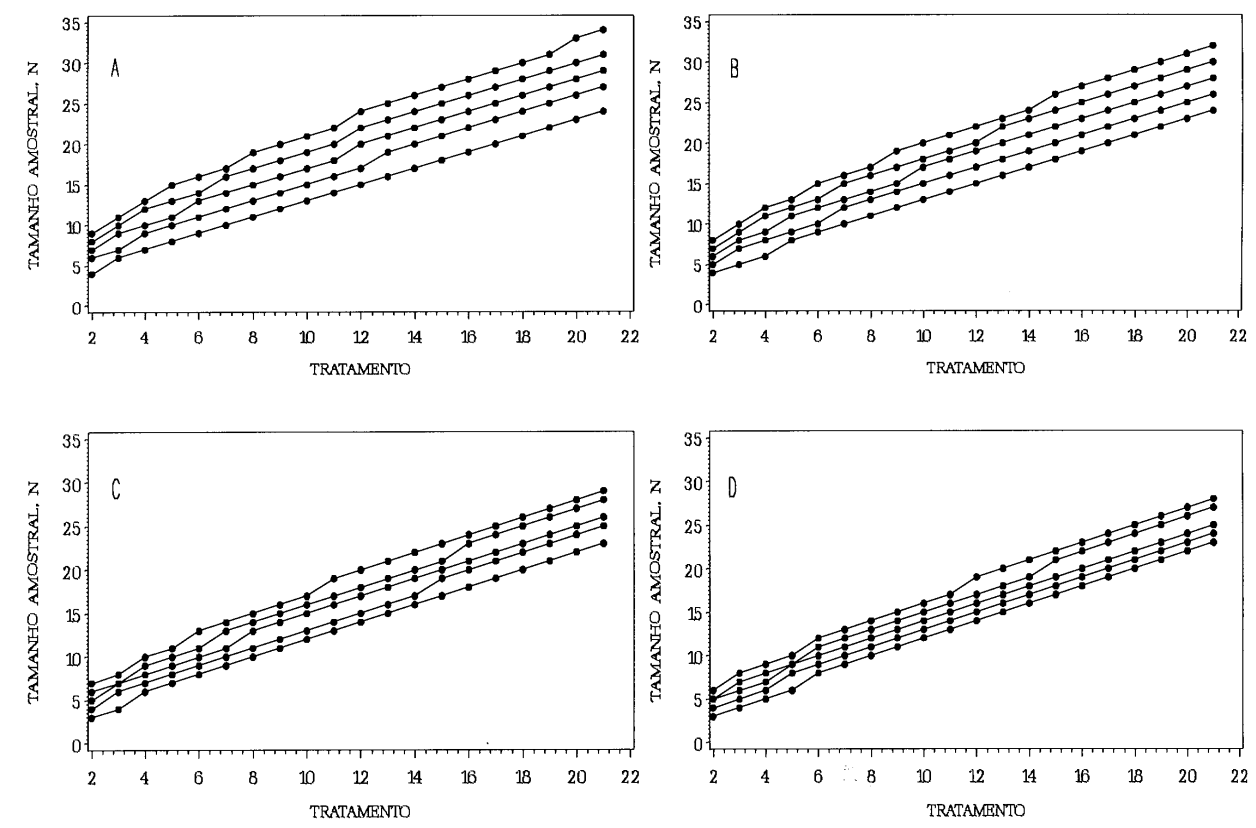

Figura 6 - Estimativas do tamanho amostral $(\mathrm{N})$ para $\Delta=2,5 \sigma \alpha=0,01$ e $\beta=0,05$ (A), $\alpha=0,01$ e $\beta=0,10$ (B), $\alpha=0,05$ e $\beta=0,05$ (C), $\alpha=0,05$ e $\beta=0,10$ (D). As linhas, em ordem decrescente de $N$, indicam valores acima de $\rho_{\text {min }}$ de 0,$1 ; 0,3 ; 0,5$; 0,7 ; e 0,9 , respectivamente.

Figure 6 - Estimates of sample size $(N)$ for $\Delta=2.5 \sigma, \alpha=.01$ and $\beta=.05(A), \alpha=.01$ and $\beta=.10(B), \alpha=.05$ and $\beta=.05(C), \alpha=.05$ and $\beta=$ .10 (D). The rows, in decreasing valies of $n$, indicate values for $\rho_{\min }$ de .1, .3, .5, .7, and .9, respectively.

$\rho_{\min }<0,3$, independentemente de $\alpha, \beta$ e do número de avaliações (tratamento), a mudança no valor de $\Delta$ de $0,5 \sigma$ para $1,0 \sigma$ implica em redução grande no valor de n; o que não se verifica quando o valor de $\Delta$ situa no intervalo de $1,0 \sigma$ (Figura 3) até 2,5 $\sigma$ (Figura 6). Verifica-se que há necessidade de amostras grandes para se detectar diferença significativa para $\Delta=0,5 \sigma$, o que não ocorre quando se considera $\alpha \Delta$ no intervalo de $1,0 \sigma$ a $2,5 \sigma$. Portanto, para $\rho_{\text {min }}<0,3$, as estimativas de $\mathrm{n}$ são mais influenciadas por variações na $\Delta$ que para valores de $\alpha$ e $\beta$. Contudo, independentemente dos valores de $\alpha, \beta$, $\rho_{\min }$ e $\Delta$, a amplitude dos valores de $\mathrm{n}$ é grande para os tratamentos considerados $(\mathrm{t}=2, \ldots, 21)$.

Observando-se as Figuras 2 a 6 e a Tabela 3, verifica-se que, para determinado valor de $\Delta$ e $\rho$ min, há necessidade de maior valor de $\mathrm{n}$ para se detectar diferença significativa entre médias sucessivas, quando o valor de a passa de 0,05 para 0,01 do que quando a potência passa de 0,90 para 0,95 . Independentemente da potência do teste, da correlação entre medidas repetidas e da $\Delta$, para se comprovar diferença significativa entre médias ao nível de $\alpha=0,01$, há necessidade de amostra cerca de $35,0 \%$ maior quando comparada a $\alpha=0,05$.

VONESH e SCHORK (1986) estudaram o tamanho amostral de três a seis condições de avaliação, sete valores de $\Delta(1,0 \sigma$ a $3,0 \sigma)$, potência do teste $(0,80$ e 0,90$)$, correlação mínima variando de 0,1 a 0,9 e a igual a 0,01 e 0,05. Foi observada redução acentuada nas estimativas de $\mathrm{n}$ quando $\Delta$ passou de $1,0 \sigma$ para $1,25 \sigma$, observando-se redução proporcionalmente menor daí por diante. Vale salientar que a eficiência da estimativa do tamanho amostral é dependente da acurácia na obtenção da matriz de variância-covariância amostral.

\section{Conclusões}

O número de animais necessário para se detectar significância entre avaliações mensais repetidas (tratamentos) de circunferência escrotal de bovinos Nelore, dos 10 aos 30 meses de idade, é bastante influenciado por diferença mínima significativa $(\Delta)$, correlação entre as medidas repetidas, erro do tipo I $(\alpha)$ e potência do teste $(1-\beta)$. 
Há necessidade de amostra grande de animais para se detectar diferença significativa, quando se considera $\Delta=0,5 \sigma$ comparada a valores de $\Delta$ no intervalo de $1,0 \sigma$ a $2,5 \sigma$.

Para determinado valor de $\Delta$, há necessidade de amostra maior de animais para se comprovar diferença significativa entre médias, quando a passa de 0,05 para 0,01 do que quando a potência altera de 0,90 para 0,95 .

Independentemente da potência do teste, da correlação entre medidas repetidas e da $\Delta$, para se comprovar diferença significativa entre médias em nível de $\alpha=0,01$, há necessidade de amostra cerca de $35,0 \%$ maior quando comparada a $\alpha=0,05$.

Independentemente da precisão com que se deseja comprovar diferença significativa entre médias sucessivas, há acréscimo praticamente linear dos valores de $\mathrm{n}$, à medida que se aumenta o número de tratamentos $(\mathrm{t}=2, \ldots, 21)$.

\section{Referências Bibliográficas}

ALENCAR, M.M., BARBOSA, P.F., BARBOSA, R.T. et al. 1993. Parâmetros genéticos para peso e circunferência escrotal em touros da raça Canchim. R. Soc. Bras. Zootec., 22(4):572-583.

ALENCAR, M.M., VIEIRA, R.C. 1989. Crescimento testicular de touros da raça Canchim. Pesq. Agrop. Bras., 24(11):1329-1333.

BOURDON, R.M., BRINKS, J.S. 1986. Scrotal circumference in yearling hereford bulls: adjustment factors, heritabilities and genetic, environmental and phenotypic relationship with growth traits. J. Anim.Sci., 62(4):958-967.

BRINKS, J.S., McLNERNEY, M.J., CHENOWETH, P.J. 1978. Relationship of age at puberty in heifers to reproductive traits in young bulls. Proceedings... Western section: Amer. Soc. Anim. Sci., 29(1):28-30.

BROWNIE, C., BOOS, D.D., OLIVER, J,H. 1990. Modifying the $\mathrm{t}$ and ANOVA $\mathrm{F}$ tests when treatment is expected to increase variability relative to controls. Biometrics, 46(1):259-266.

COULTER, G.H., MAPLETOFT, R.J., KOZUB, G.C. et al. 1987. Scrotal circumference of two-year-old bulls of several beef breeds. Theriogenology, 27(2):485-491.

CULLIS, B.R., McGILCHRIST, C.A. 1990. A model for the analysis of growth data from designed experiments. Biometrics, 46(1):131-142.

ELER, J.P., FERRAZ, J.B.S., SILVA, P.R. 1996 Parâmetros genéticos de escores visuais e circunferência escrotal em bovinos da raça Nelore. In: SEMINÁRIO NACIONAL DE REVISÃO DE CRITÉRIOS DE JULGAMENTO E SELEÇÃO EM GADO DE CORTE, 1996, Uberaba. Anais... Uberaba: ABCZ , 4p.

FELICIANO SILVA, A.E.D., DODE, M.A., PORTO, J.A. 1991. Estacionalidade na atividade sexual de machos bovinos Nelore e mestiços Fleckvie e Chianina x Nelore. Pesq. Agrop. Bras., 26(10):1745-1750.

FREITAS, A.R., SILVA, A.E.D.F., DOBRE, M.N., SILVA, M.M.U. Taxa de crescimento da circunferência escrotal de machos nelore. In: REUNIÃO ANUAL DA SOCIEDADE BRASILEIRA DE ZOOTECNIA, 34, 1997. Juiz de Fora. Anais... Juiz de Fora: SBZ, 1997.

GEARY, D.N. 1989. Modelling the covariance structure of repeated measurements. Biometrics, 45(4):1183-1195.

HARDISON, C.D., QUADE, D., LANGSTON, R.E. 1983. Nine functions for probability distributions. In: SAS Institute Inc. SUGI Supplemental Library User's Guide. Cary: SAS Institute Inc, p.229-236.

KING, R.G., KRESS, D.D., ANDERSON, D.C. 1983. Genetic parametrs in hereford for puberty in heifers and scrotal circumference in bulls. Montana State University, Bozemen. Montana Agricultural Experiment Station, Havre. 34(1):11-13.

KRIESE, L.A., BERTRAND, J.K., BENYSHEK, L.L. 1991. Age adjustment factors, heritabilities and genetic correlations for scrotal circumference and related growth traits in hereford and brangus bulls. J. Anim. Sci., 69(2):478-489.

LUNSTRA, D.D., GREGORY, K.E., CUNDIFF, L.V. 1988. Heritability estimates and adjustmen for the effects of bull age of dam on yearling testicular size in breeds of bulls. Theriogenology, 30(1):127-136.

MANSOUR, H., JENSEN, E.L., JOHNSON, L.P. 1991. Analysis of covariance structure of repeated measurements in holstein conformation traits. J. Dairy. Sci., 74(8):2757-2766.

MOURA, A.A.A., ERICKSON, B. Estimativa das relações entre peso testicular aos 12 meses e níveis hormonais entre 2 e 12 meses em tourosda raça angus. In: REUNIÃO ANUAL DA SOCIEDADE BRASILEIRA DE ZOOTECNIA, 33, 1996, Fortaleza. Anais...Fortaleza: SBZ, p.341-343, 1996.

PINTO, P.A. O perímetro escrotal como critério de seleção em bovinos Nelore. In: SEMINÁRIO NACIONAL DE REVISÃO DE CRITÉRIOS DE JULGAMENTO E SELEÇÃO EM GADO DE CORTE, 1996, Uberaba. Anais... Uberaba: ABCZ, 7p.1996.

PRATT, S.L., SPITZER, J.C. WEBSTER, H.W. et al. 1991. Comparisom of methods for predicting yearling scrotal circumference and correlations of scrotal circumference to growth traits in beef bulls. J. Anim.Sci., 69(7):2711-2720.

QUIRINO, C.R., BERGMANN, J.A.G., OLIVEIRA, S.M.P. 1996. Taxa de crescimento relativo como critério para avaliar o desenvolvimento ponderal e testicular de animais nelore. In: REUNIÃO ANUAL DA SOCIEDADE BRASILEIRA DE ZOOTECNIA, 33, 1996, Fortaleza. Anais... Fortaleza: SBZ, p.184-185.

SAS INSTITUTE. 1993. SAS/STAT User's guide: statistics, versão 6 , v.2, 4. ed. Cary.

SCHEFFÉ, A. 1959. The analysis of variance. John Wiley. 477p.

TOELLE, V.D., ROBISON, O.W. 1985. Estimates of genetic correlations between testicular measurements and female reproductive traits in cattle. J. Anim.Sci., 60(1):89-100.

URIBE, H. 1996. Random regressions in animal breeding. Workshop: Melhoramento de gado de corte na EMBRAPA. Campo Grande - MS. p.1-20, Nov. 17 to 26.

VONESH, E.F., SCHORK, M.A. 1986. Sample size analysis of repeated measurement. Biometrics, 42(3):601-610.

WERRE, J.F., BRINKS, J.S. 1986. Relationship of age at puberty with growth and subsequent productivity in beef heifers. Proceedings... Western section: Amer. Soc. Anim. Sci., 37(2):300-303.

Recebido em: 11/08/97

Aceito em: 27/08/98 